\title{
Proposta de Plantas Alternativas para Ensino de Sistemas De Controle Utilizando Conceitos de Óptica Adaptativa
}

DOI: 10.37702/2175-957X.COBENGE.2021.3441

Victor André Houri e Costa - costa.victor@usp.br

Universidade de São Paulo

Rua Viamão 1139

30431-253 - Belo Horizonte - MG

Wesley Beccaro - wesley@Ime.usp.br

Universidade de São Paulo

Av. Prof. Luciano Gualberto 158

05508-010 - São Paulo - SP

Resumo: Durante o ensino da disciplina de controle, são apresentados aos alunos diversos exemplos de plantas com o objetivo de explicitar os conceitos aprendidos em aplicações reais. Essas plantas, normalmente, são termodinâmicas ou eletromecânicas e apresentam dinâmicas de primeira ou de segunda ordem. Com a apresentação de plantas que não são comumente trabalhadas em sala de aula, pode-se de expandir a capacidade dos alunos em aplicar os conceitos de modelagem, de simulação, de projeto e de sintonia de controladores. Este trabalho propõe três experimentos em uma planta de óptica adaptativa (AO). O primeiro experimento consiste na criação e na configuração de blocos simuladores de um sistema de óptica adaptativa. O segundo experimento consiste no desenvolvimento de um controlador de óptica adaptativa através da simulação de um controlador de ação Integral pura para a planta já configurada. O último experimento consiste na aplicação de um controlador de ação Proporcional-Integral. Durantes todas experiências são gerados diagramas e gráficos que permitem a discussão de maneira quantitativa e qualitativa do desempenho dos controladores simulados pelos alunos. Desta forma, são introduzidos os conceitos de instrumentação astronômica, permitindo aos alunos o exercício de conceitos relacionados à teoria de controle em um contexto inovador.

Palavras-chave: Óptica Adaptativa. Instrumentação Astronômica. Controle de Processos. Controlador PID. Ensino de Engenharia. 


\section{Proposta de Plantas Alternativas para Ensino de Sistemas De Controle Utilizando Conceitos de Óptica Adaptativa}

\section{INTRODUÇÃO}

O ensino de sistemas de controle oferece aos alunos tanto uma base matemática sólida (OGATA, 2010), como dá suporte prático para que consigam construir modelos matemáticos de sistemas reais e o projeto de controladores (NISE, 2012). Um grande desafio desta disciplina é garantir que os alunos consigam aplicar o conhecimento teórico em situações práticas durante sua trajetória profissional (FACCIN, 2004) (RAPKIEWICZ et al., 2016). Neste sentido, estando de acordo com o que se espera de um egresso do curso de Engenharia, conforme perfil traçado pela diretriz curricular nacional dos cursos de graduação em Engenharia, que tem como base garantir que estes profissionais consigam pesquisar, formular e atuar perante os problemas de suas áreas (MEC, 2019).

Este trabalho propõe o desenvolvimento de experimentos que permitam aos alunos de graduação e de pós-graduação compreender os conceitos de óptica adaptativa (AO) no contexto da instrumentação astronômica (REEVES, 2016) (CONAN; CORREIA, 2014). Além disso, através desse contato com uma planta não tradicional, distinta daquelas usualmente vistas em disciplinas de controle básico ou avançado (sistemas de controle de nível, vazão, temperatura, velocidade, entre outros), permite-se ao aluno maior contextualização das aplicações de modelagem e controle em áreas de pesquisa avançada. Por meio destes experimentos, são trabalhados conceitos fundamentais de controle, dentre eles: modelagem de sistemas complexos, projeto e sintonia de controladores e avaliação de métricas de saída. Com isso, os alunos poderão compreender os fenômenos relacionados à óptica adaptativa em um ambiente de aprendizagem experimental.

Este artigo está estruturado da seguinte forma: a seção 2 apresentada os conceitos teóricos básicos de uma planta de óptica adaptativa. A seção 3 apresenta os materiais e métodos utilizados na proposta de desenvolvimento das experiências destacando os softwares e as bibliotecas contendo os modelos de óptica adaptativa e controle utilizados. Nesta mesma seção são descritos os três experimentos propostos, através de um roteiro mínimo com a indicação de uma teoria básica e objetivos de cada experimento. A seção 4 apresenta alguns resultados que podem ser alcançados através dos experimentos, servindo de referência ao professor. Por fim, as considerações finais e conclusões são descritas na seção 5 .

\section{CONCEITOS DE ÓPTICA ADAPTATIVA}

\subsection{Estrutura Típica de uma Planta de Óptica Adaptativa}

A óptica adaptativa consiste em uma técnica de controle em malha fechada para corrigir uma frente de onda que possui algum tipo de perturbação devido à presença de turbulências atmosféricas. Desta forma, possibilitando o aumento da resolução efetiva dos telescópios baseados em solo (FRIED, 1966). Esse tipo de sistema possui, dentre outros elementos, um espelho deformável (DM) e um sensor de frente de onda (WFS).

A operação da malha fechada de controle se dá por meio da adoção de uma fonte de luz de referência, que possui características de perturbação conhecidas. Quando essa fonte de luz é uma estrela natural ela é chamada de Natural Guide Star (NGS) e quando ela é um ponto de Laser projetado artificialmente no céu, é chamada de Laser Guide Star 
(LGS) (OLIVIER; MAX,1994). O caminho óptico da frente de onda gerada por esta fonte, independentemente de sua natureza, pode ser exemplificado na Figura 1.

Figura 1 - Diagrama de montagem de um sistema de óptica adaptativa com espelho deformável e sensor de frente de onda.

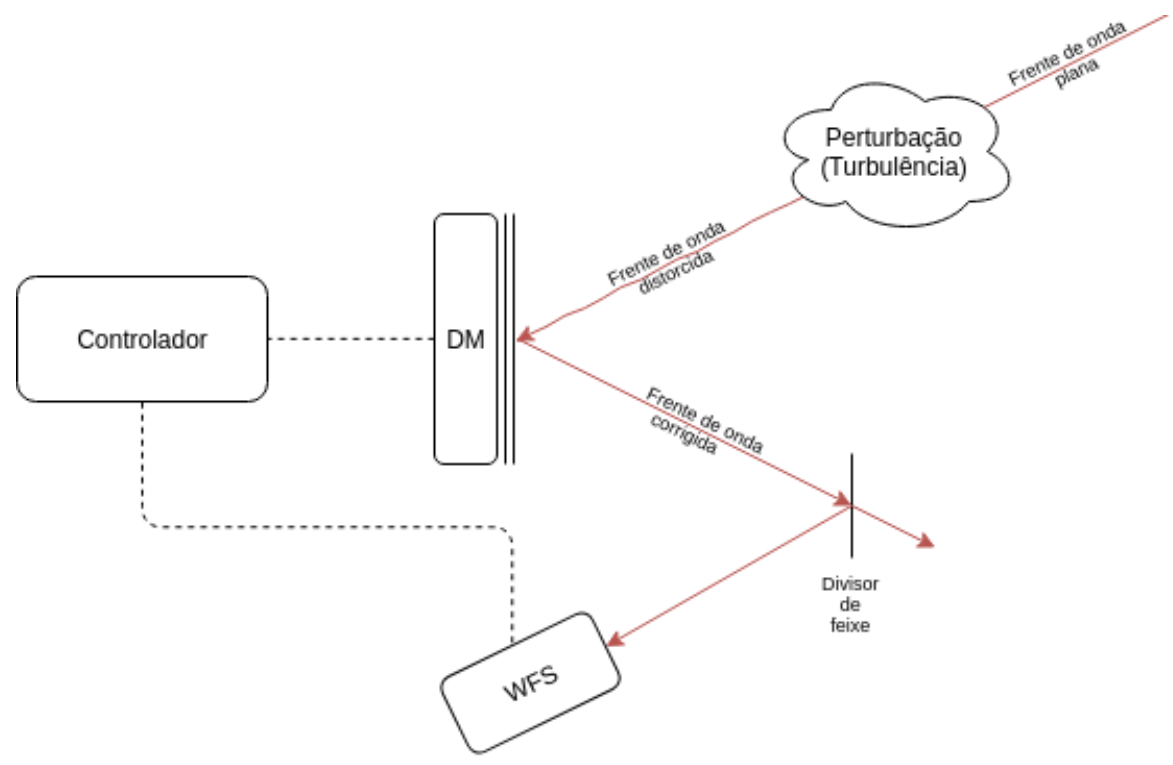

Fonte: Autores (2021).

Uma adaptação da montagem típica de um sistema de óptica adaptativa para instrumentação astronômica sem a necessidade da construção de um telescópio inteiro pode ser realizada utilizando um Laser cuja emissão passa por uma lente termicamente tratada a fim de gerar uma perturbação na frente de onda. A Figura 2 apresenta um diagrama dessa montagem adaptada para bancada (MAUCH, 2016).

Figura 2 - Diagrama de montagem de um sistema de óptica adaptativa em bancada.

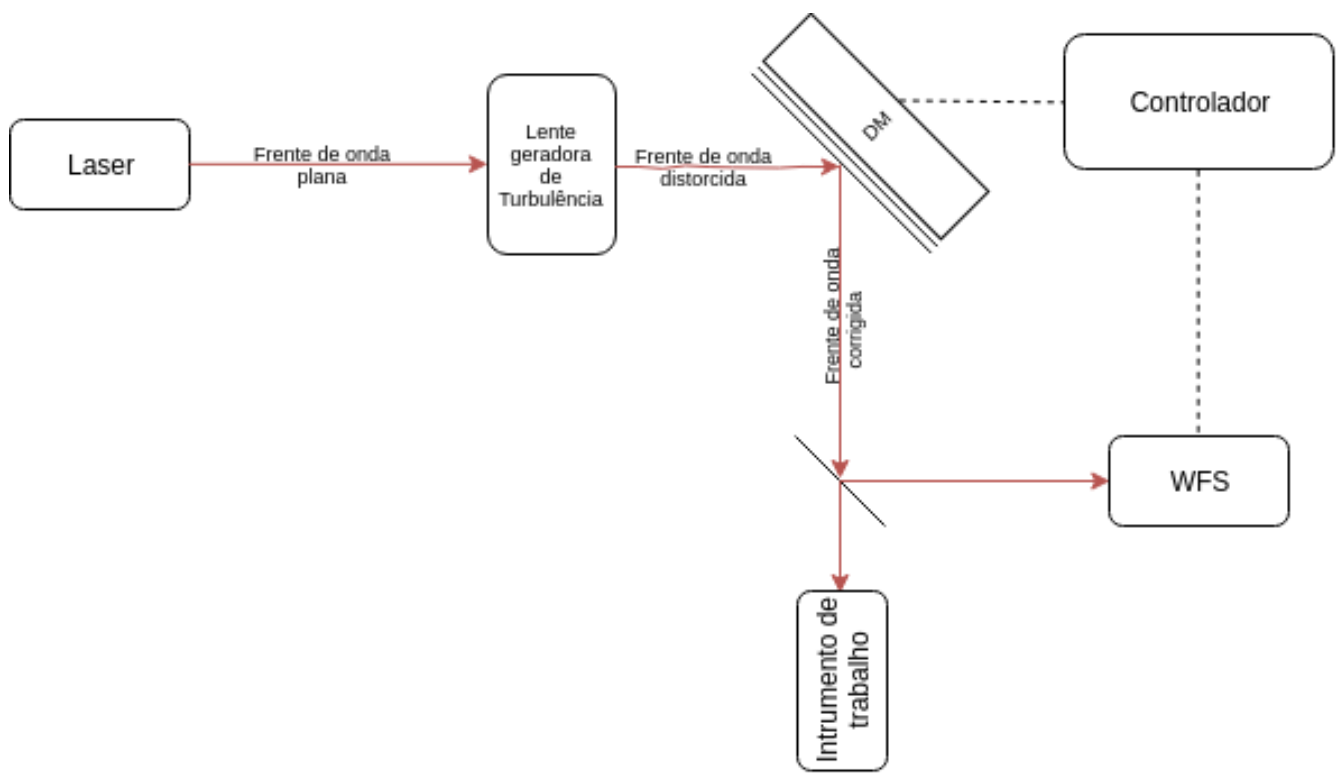

Fonte: Autores (2021). 


\section{ADAPTATIVA}

\section{EXPERIMENTOS DE MODELAGEM E CONTROLE EM ÓPTICA}

A tecnologia final utilizada para implementar sistemas de $A O$ normalmente não permite uma implementação direta da malha de controle sem que antes diversos projetos de controladores e simulações deste sistema tenham sido executados. A fim de auxiliar o projeto de controladores de óptica adaptativa, diversos modelos de turbulência atmosférica e de óptica adaptativa foram desenvolvidos ao longo dos anos. O YAO, desenvolvido em Yorick, é um proeminente e clássico simulador de AO. Atualmente, outros simuladores vêm ganhando atenção, como o Simulation 'Optique Adaptative' with Python (SOAPY) (REEVES, 2016), desenvolvido em Python, e o Object-Oriented, Matlab \& Adaptive Optics Toolbox (OOMAO), que é desenvolvido em MATLAB (CONAN; CORREIA, 2014). Nos experimentos propostos é utilizado o OOMAO, que consiste em um toolkit de óptica adaptativa que utiliza os preceitos de orientação a objetos e que possui 5 classes principais: source, atmosphere, telescope, shackHartmann (sensor de frente de onda) e deformmableMirror.

No primeiro experimento os métodos de configuração de entrada do OOMAO são apresentados aos alunos. Nele é realizada uma introdução aos modelos do framework, bem como a configuração das fontes, condições atmosféricas, dimensões e características do telescópio (sensor de frente de onda e espelho deformável). Neste experimento o foco é apresentar as saídas em malha aberta. No segundo experimento, uma vez que os alunos já possuem familiaridade com os modelos, é discutida a implementação de um laço de realimentação utilizando controle Integral (I), amplamente utilizado em óptica adaptativa. Nesta experiência se avaliam comparativamente as métricas de saída do sistema em malha aberta em comparação com o sistema em malha fechada. No último experimento, solicita-se aos alunos projetarem e sintonizarem um controlador Proporcional-Integral (PI) a fim de analisar os resultados obtidos em malha aberta e em malha fechada com os controladores I e PI.

\subsection{Experimento 1: Modelos de Óptica Adaptativa: Introdução ao Framework de Modelagem e Controle em Óptica Adaptativa OOMAO}

Uma vez apresentado o framework, cada bloco é configurado de acordo com uma especificação proposta, conforme descrito nos subitens a seguir.

\subsubsection{Atmosfera}

A classe atmosfera simula numericamente o meio por onde caminha o feixe de luz proveniente da fonte. Nesta classe é modelada a turbulência que gera as deformações de fase do sistema. Essa turbulência é gerada por variações aleatórias no índice de refração do ar. No OOMAO existem dois modelos implementados, o de Kolmogorov e o de von Kárman. No construtor da classe atmosphere, são passados os parâmetros do comprimento de onda a ser analisado, o parâmetro de Fried $\left(r_{0}\right)$ e o outer scale da turbulência. Além disso, são configurados outros parâmetros atmosféricos, como, por exemplo, velocidade e direção do vento, além de altitude das turbulências. Pede-se aos alunos, para um exemplo de aplicação real, que instanciem um objeto do tipo atmosfera, que simulará turbulências na banda I, com um ro de $0,14 \mathrm{~m}$, um outer scale (L0) de $30 \mathrm{~m}$, operando nas altitudes de $\left\{\begin{array}{lllll}0 & 1 & 2 & 4\end{array}\right\} \mathrm{km}$, com contribuições parciais de turbulência de

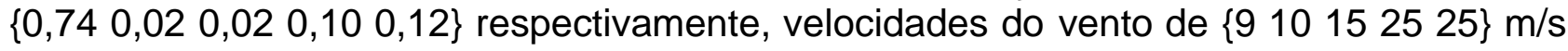


e direção do vento em $\{0 \pi / 2 \pi 3 \pi / 22 \pi\}$. A implementação final dessa instância é indicada na Figura 3.

Figura 3 - Implementação da classe atmosphere.

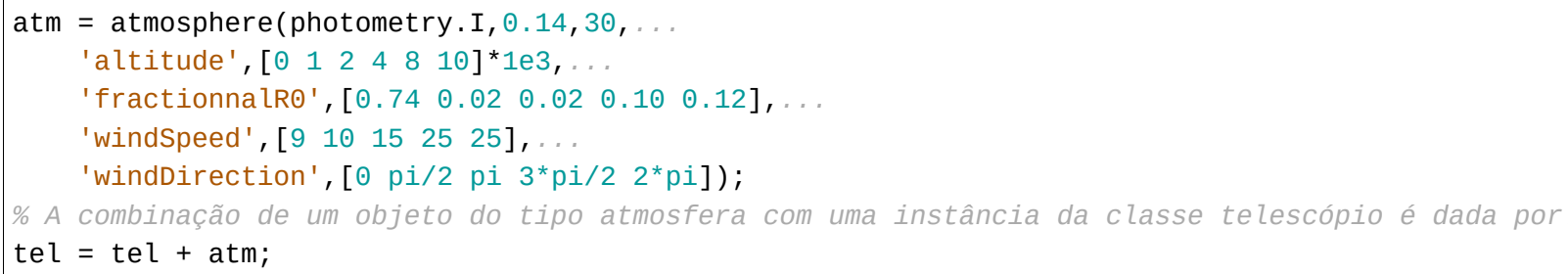

Fonte: Autores (2021).

\subsubsection{Telescópio}

A classe telescópio possui duas tarefas, definir os parâmetros do telescópio e calcular a turbulência simulada numericamente, conforme configurada na classe atmosfera. No construtor da classe telescope, é passado o diâmetro da pupila do telescópio. Além disso, são configurados os demais dados do telescópio, como, por exemplo, campo de visão, resolução, frequência de amostragem e aberração. Pede-se aos alunos que instanciem um objeto do tipo telescope com um diâmetro de $4,1 \mathrm{~m}$, um campo de visão de 3 arco minutos, uma resolução de 128 Pixels, um período de amostragem de $2 \mathrm{~ms}$, e uma razão de obstrução de 0,228. A implementação final desse bloco é descrita na Figura 4.

Figura 4 - Implementação da classe telescope.

tel $=$ telescope $(4.1$, ' fieldofViewInArcMin ' , 3,

'resolution', 128, 'samplingTime' ,2e-3, 'obstructionRatio', 0.228); \% A propagação da frente de onda entre a fonte e o telescópio é: ngs=ngs . * +tel;

Fonte: Autores (2021).

\subsubsection{Fonte}

A classe de fonte permite a simulação da fonte de luz que é usada como referência a um sistema de óptica adaptativa para instrumentação astronômica. Os dois métodos mais comuns estão disponíveis no OOMAO: NGS e o LGS. A instância do objeto source é obtida por meio de seu construtor e é esperado que os alunos instanciem dois objetos desse tipo. O primeiro, uma fonte de calibração operando no ultravioleta e o segundo, a fonte utilizada no controle, também operando no ultravioleta, mas com uma altitude de 7062 m e uma magnitude de 3,5. A implementação final é dada na Figura 5.

Figura 5 - Implementação da classe source.

ngs $=$ source ('wavelength', photometry. $U)$;

lgs = source( 'wavelength', photometry.U ,' height' ,7062, 'magnitude' , 3.5);

Fonte: Autores (2021).

\subsubsection{Sensor de Frente de Onda}

A classe do sensor de frente de onda instância um objeto do tipo Shack-Hartmann, o tipo mais comum de sensor de frente de onda para sistemas de óptica adaptativa, que 
simula a aquisição da frente de onda em uma matriz de microlentes por meio do cálculo de um mapa de intensidades. No construtor da classe shackHartmann, é passado o número de lenslets, a resolução do detector em pixels e a razão mínima de luz. Os alunos deverão criar uma instância desse tipo com uma matriz de 16 × 16 lenslets, $128 \times 128$ pixels e uma razão mínima de luz de 0,75. A implementação está indicada na Figura 6 :

Figura 6 - Implementação da classe shackHartmann.

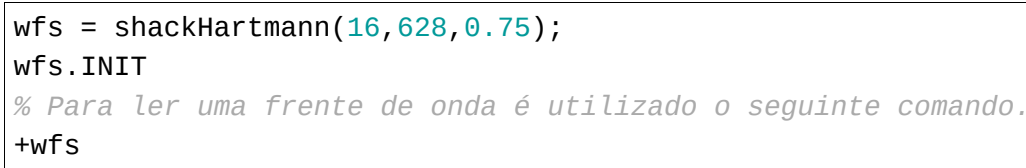

Fonte: Autores (2021).

\subsubsection{Espelho Deformável}

Conforme descrito anteriormente, o espelho deformável é o elemento ativo e o atuador de um sistema de óptica adaptativa. No construtor da classe deformableMirror é configurado o número de atuadores, além dos demais dados do espelho deformável, como, por exemplo, resolução, modos e função de influência. Pede-se aos alunos que criem um objeto deste tipo com $17 \times 17$ atuadores, função de influência monotônica, acoplamento mecânico de $20 \%$ e resolução de 128 Pixels. Também é necessário que os alunos informem ao espelho quais atuadores são de fato utilizados, isso pode ser feito passando a máscara da região de interesse do sensor de frente de onda. A Figura 7 apresenta a implementação.

Figura 7 - Implementação da classe deformableMirror e métodos de propagação e atualização dos

coeficientes do espelho deformável.

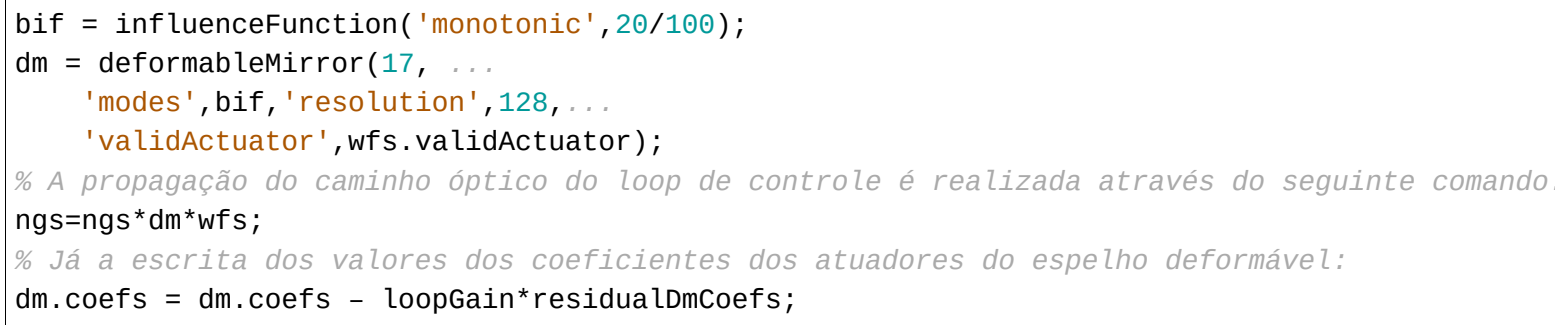

Fonte: Autores (2021).

\subsubsection{Levantamento da Matriz de Influência}

Antes da execução da malha de controle de óptica adaptativa, é necessário que se faça a determinação, normalmente empírica, da matriz de influência DM-WFS. Essa matriz de ordem $2 n \times m$, sendo que $n$ é o número de microlentes do sensor de frente de onda e $m$ é o número de atuadores do espelho deformável. Experimentalmente, essa matriz é determinada por meio da atuação de cada um dos atuadores um a um e pela medição dos deslocamentos que isso proporciona no sensor de frente de onda. Posteriormente essa matriz de influência é submetida a uma pseudo-inversão para que possa ser utilizada no controle. No OOMAO, todo esse procedimento pode ser feito por meio de uma chamada da função calibration, conforme indicado na Figura 8. 
Figura 8 - Uso da função calibration.

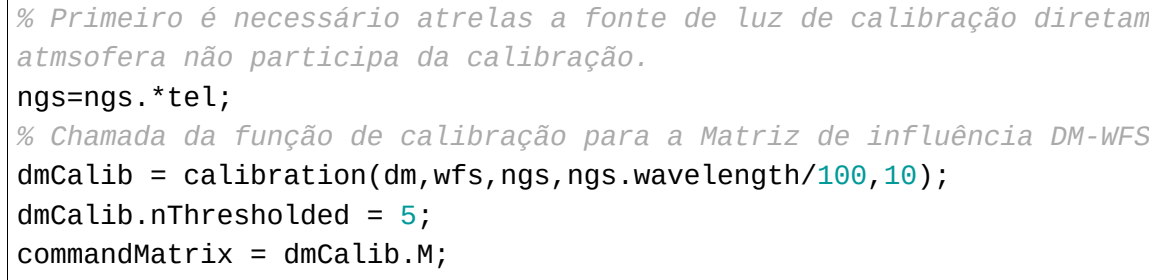

Fonte: Autores (2021).

\subsection{Experimento 2: Implementação de um Controle Integral}

O diagrama de blocos de controle de um sistema de óptica adaptativa está descrito no diagrama da Figura $9 . G_{p}$ representa o processo que adiciona a turbulência atmosférica no sistema, $G_{c}$ é o controlador, $C$ é a saída do sensor de frente de onda comparada com a referência $\overline{\mathbf{C}}$ (obtida através de uma frente de onda plana), $\mathbf{H}$ é a matriz de influência, $\mathbf{U}$ é a ação de controle e $\Phi$ é a fase residual. A estratégia de controle mais comum utilizada em sistemas de óptica adaptativa é a de ação integral pura. O controlador, portanto, é um integrador discreto com um ganho.

Figura 9 - Diagrama de blocos de uma malha de controle típica de óptica adaptativa.

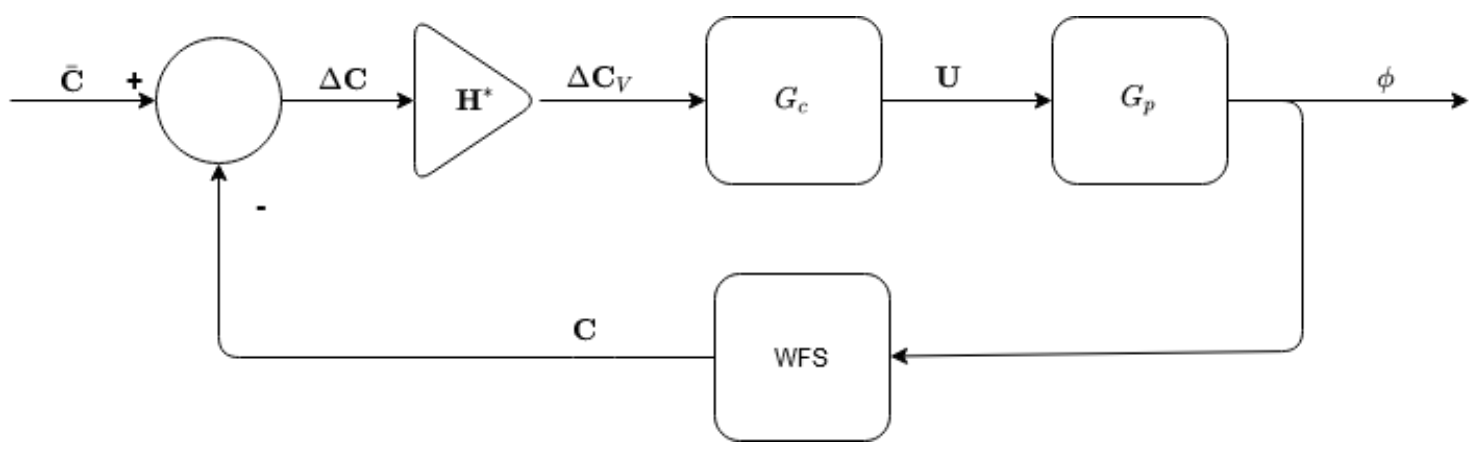

Fonte: Adaptado de (GONZALEZ-CAVA et al., 2017).

O OOMAO não possui nativamente uma interface para sua simulação em linguagem gráfica, como, por exemplo, a do Simulink. A sua malha de controle, portanto, deve ser implementada diretamente em linha de código. Antes da malha fechada propriamente dita, uma estrutura de preparação deve ser feita, garantindo a correta propagação da frente de onda por todos os blocos criados pelos alunos e inicializando os vetores que armazenarão os dados do controle. A implementação dessa infraestrutura, que os alunos devem implementar ou, a depender da estratégia do professor, ser fornecida, é apresentada na Figura 10.

A simulação da malha fechada pode ser feita de acordo com a implementação realizada na Figura 11. Após a simulação da malha fechada, é necessário que os alunos gerem os gráficos que exibam as métricas de desempenho de controle. Diversas métricas podem ser extraídas a partir da simulação, mas as mais básicas do OOMAO são: valor efetivo da frente de onda, desvios do centroide de frente de onda e ação de controle. Com base nos dados capturados pelas variáveis auxiliares no passo anterior, os alunos deveram criar gráficos e digramas que exibam as métricas de desempenho de um sistema de óptica adaptativa. A Figura 12 apresenta uma implementação desses gráficos considerando o código apresentado na Figura 11. 
Figura 10 - Inicialização das variáveis.

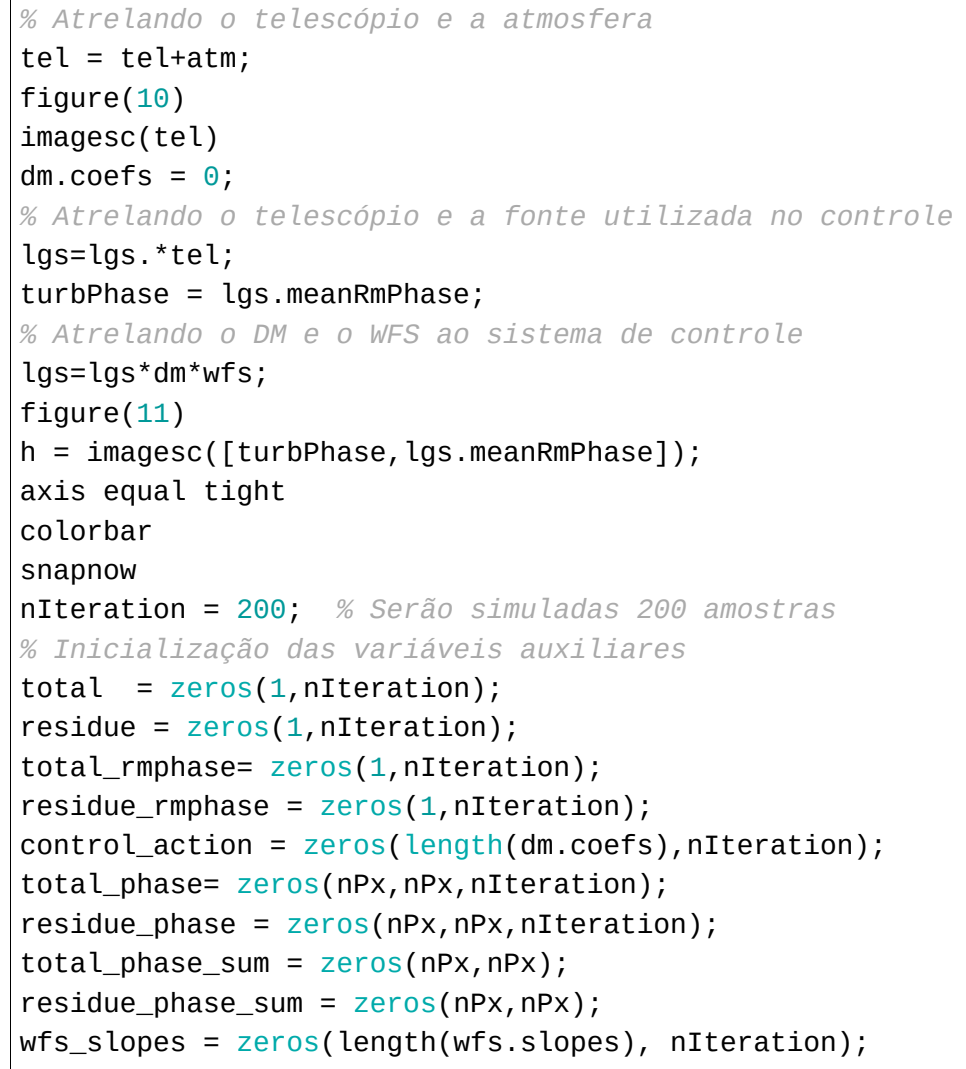

\section{Fonte: Autores (2021).}

Figura 11 - Implementação da malha fechada.

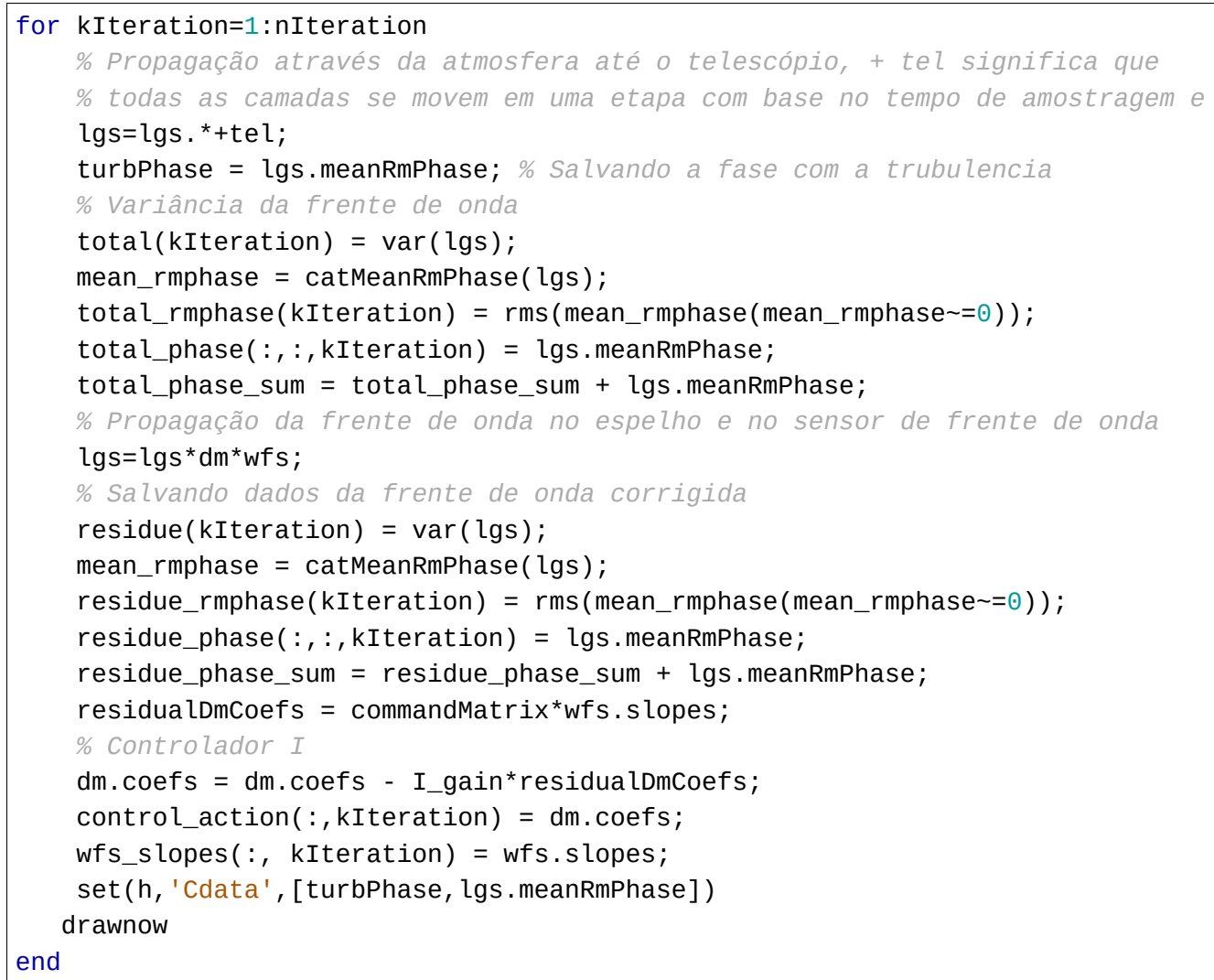

Fonte: Autores (2021). 
Figura 12: Código fonte para geração dos gráficos que exibem as métricas de controle: valor efetivo de frente de onda, ação de controle e desvios dos centroides do WFS.

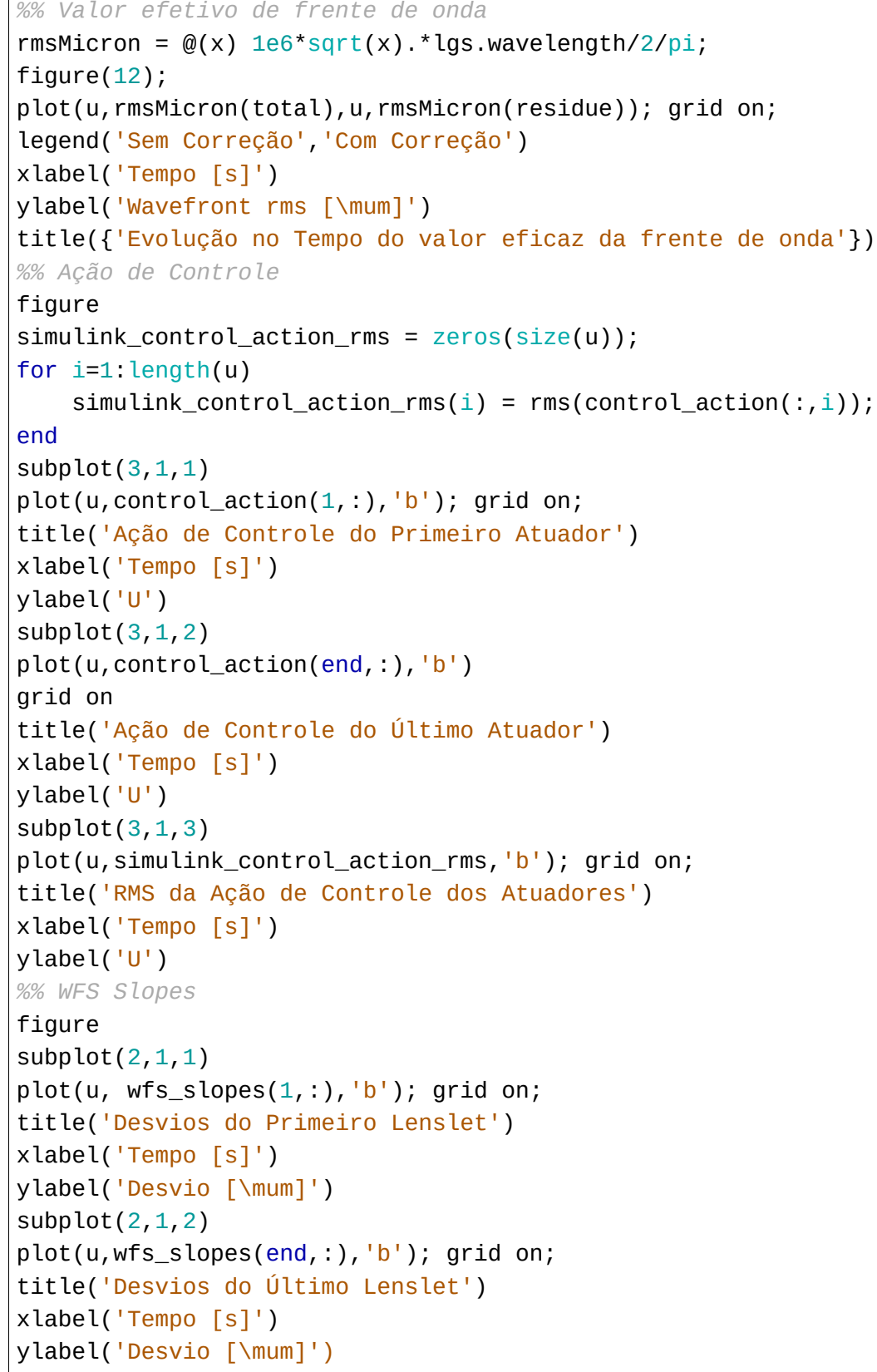

Fonte: Autores (2021).

\subsection{Experimento 3: Implementação de um Controle Proporcional-Integral}

O controlador PI também deve ser implementado em linha de código. Embora exista várias implementações distintas de tal estratégia de controle, o controlador PI paralelo é o mais simples de todos. O professor pode aproveitar essa oportunidade para discutir as diversas formas de se discretizar e de se implementar um PI. Pede-se aos alunos que modifiquem o código do experimento anterior para que ele passe a adotar essa estratégia de controle e que eles sintonizem o novo controlador. A implementação de um PI paralelo em linha de código, compatível com o código apresentado na Figura 11 está presente na Figura 13. Os alunos também deverão gerar as análises de avaliação de desempenho para essa estratégia de controle. 
Figura 13 - Implementação da Malha Fechada em um PI.

$I=d m$. coefs - I_gain*residualDmCoefs;

dm. coefs $=$ P_gain* $(-$ residualDmCoefs $)+I ;$

Fonte: Autores (2021).

\section{$4 \quad$ PRINCIPAIS RESULTADOS DOS EXPERIMENTOS}

Diversos gráficos e diagramas podem ser gerados durante os experimentos. A Figura 14 apresenta o gráfico em que é possível observar o valor efetivo da frente de onda para o sistema em malha aberta (sem correção) e utilizando realimentação através controlador I (com correção). Esse gráfico é gerado a partir dos códigos apresentados na Figura 12. Essa medida representa o quanto a fase de uma frente de onda está modificada, sendo que zero, condição ideal, representa uma frente de onda plana. Observa-se que há uma redução significativa no valor efetivo da frente de onda com a correção, partindo de uma média de $0,8 \mu \mathrm{m}$ para o sistema sem correção, para aproximadamente 0,3 $\mu \mathrm{m}$ (redução de 60\%).

Figura 14: Evolução no tempo do valor eficaz da frente de onda para o sistema em malha aberta (sem correção) com utilizando controlador I (com correção).

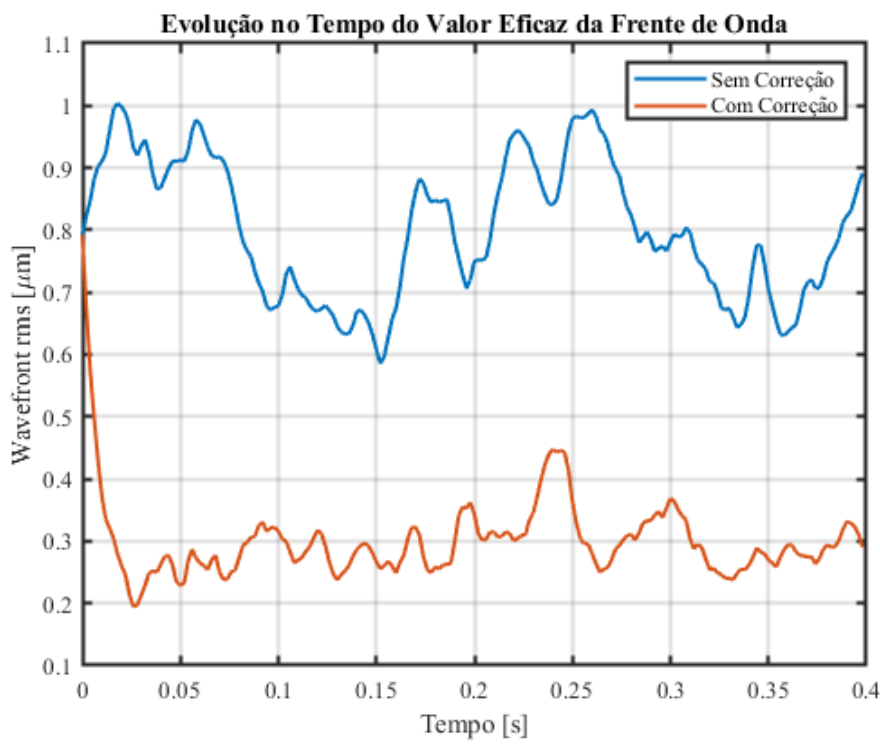

Fonte: Autores (2021).

A título de comparação, durante os experimentos os alunos devem explorar a estratégia PI que produz gráficos equivalentes ao apresentado anteriormente. A Figura 15 apresenta o gráfico em que é possível observar o valor efetivo da frente de onda em malha aberta (sem correção) e com o controlador PI (com correção). É possível observar que a média do valor da frente de onda com correção, aproximadamente 0,18 $\mu \mathrm{m}$, representa uma melhoria de $75 \%$ em relação ao sistema sem correção. Além disso, é importante indicar aos alunos o melhor desempenho do controlador PI em relação ao I, que, como já dito, é uma estratégia de controle amplamente utilizada nestes sistemas. 
Figura 15. Evolução no tempo do valor eficaz da frente de onda para o sistema em malha aberta (sem correção) com utilizando controlador PI (com correção).

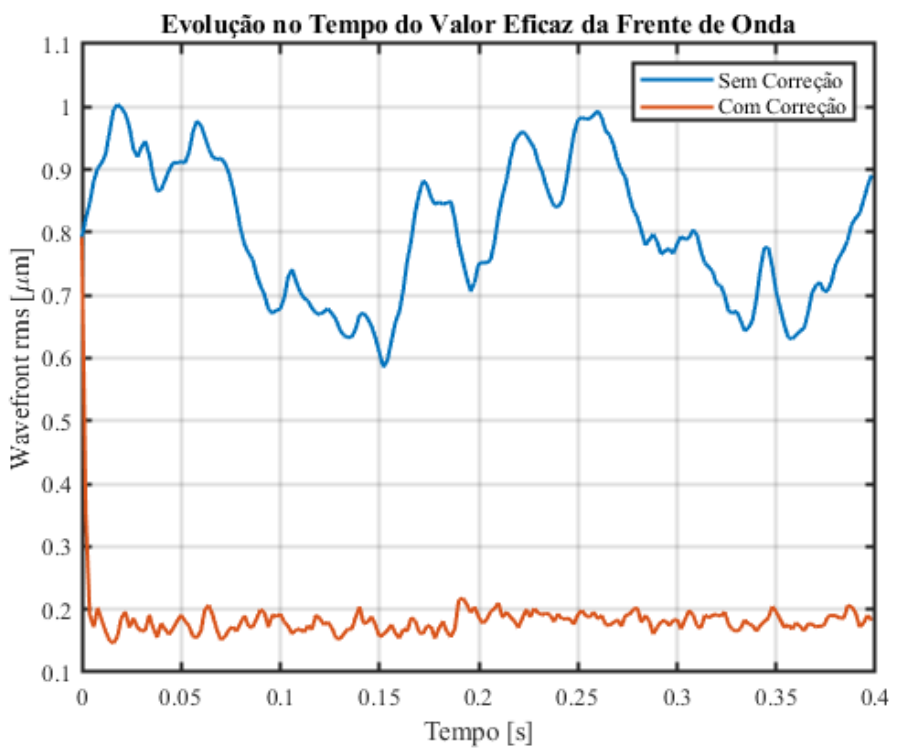

Fonte: Autores (2021).

Outros gráficos também podem ser discutidos com os alunos durante as experiências. A Figura 16 apresenta a ação de controle do primeiro e do último atuador, além do valor efetivo de todos os atuadores para o controlador I (conforme Figura 9). Salienta-se aos alunos a importância de monitorar a ação de controle durante a atuação do sistema para que se garante entrada de sinais com amplitudes realizáveis pelo atuador, sem que haja, por exemplo, saturações.

Figura 16: Ação de Controle do primeiro e do último atuador, e o valor efetivo de todos os atuadores da simulação de uma malha com o controlador Integral.
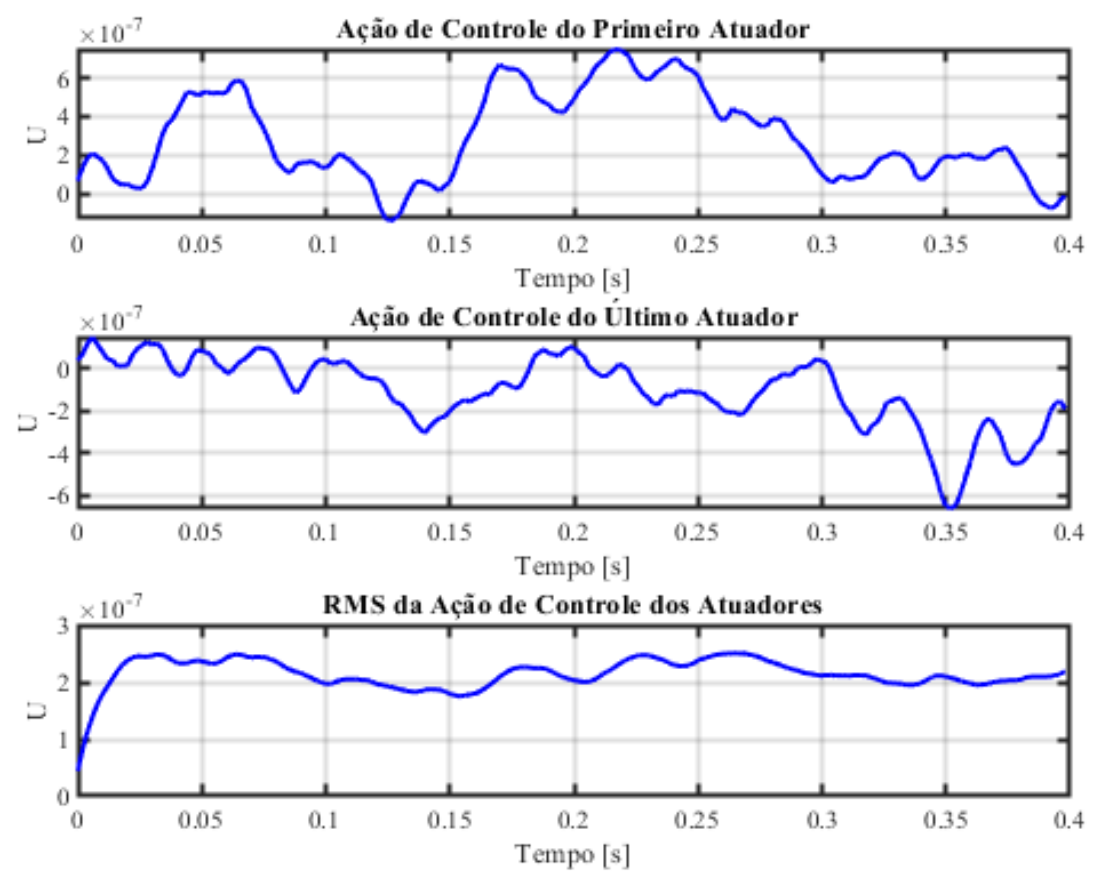

Fonte: Autores (2021). 
A Figura 17 apresenta uma representação esquemática da fase observada pelo telescópio na extensão do espelho principal. Este diagrama é obtido através dos códigos apresentados na Figura 11 e é atualizado em tempo real de simulação à medida que o sistema evoluiu ao longo do tempo, permitindo aos alunos uma visualização intuitiva do comportamento do sistema. No diagrama estão representados dois espelhos que indicam, respectivamente, a fase da frente de onda observada pelo telescópio sem correção (à esquerda) e com correção (à direita). É importante indicar aos alunos que após a atuação da realimentação, os valores de fase ficam mais próximos a zero, reduzindo a aberração proporcionada pela turbulência atmosférica e possibilitando um melhor imageamento.

Figura 17: Fase observada pelo telescópio na extensão do espelho principal. À esquerda em malha aberta e à direita com o controlador Integral.

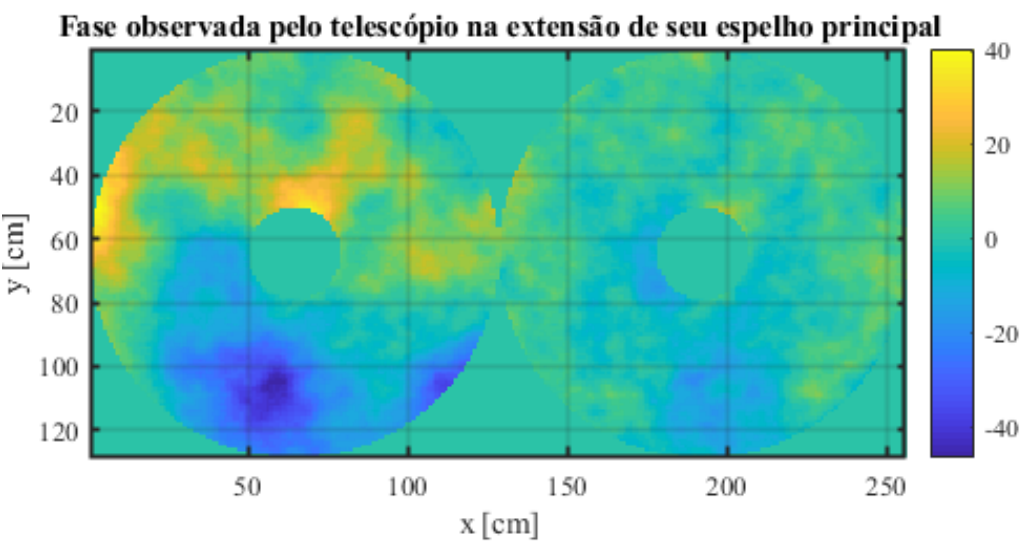

Fonte: Autores (2021).

\section{CONCLUSÕES}

Neste trabalho foram propostos três experimentos de sistemas de óptica adaptativa que permitem ampliar o escopo das plantas oferecidas aos alunos nos cursos de sistemas de controle. Os experimentos apresentam os modelos implementados no MATLAB que permitem explorar os parâmetros de projeto de sistemas de óptica adaptativa, além de estratégias de controle. No trabalho é apresentada a biblioteca OOMAO utilizada como simulador de óptica adaptativa e amplamente aplicada pela comunidade de astronomia para projetos de telescópios. Também são discutidas questões relativas à instrumentação astronômica e são introduzidos conceitos, tais como o fenômeno de Seeing, turbulência atmosférica, fontes de luz de referência, e a aplicação de espelhos deformáveis e sensores de frente de onda. Desta forma, espera-se encorajar os alunos no emprego da teoria de sistemas de controle em aplicações que usualmente estão foram do escopo da disciplina.

\section{REFERÊNCIAS}

CONAN, R.; CORREIA, C., Object-oriented MATLAB adaptive optics toolbox. Adaptive Optics Systems IV, v. 9148, p. 2066 - 2082, 2014.

FACCIN, F. Abordagem Inovadora no Projeto de Controladores PID. Dissertação de Mestrado em Engenharia Química da Universidade Federal do Rio Grande do Sul, 2004. 
FRIED, D. L. Optical Resolution Through a Randomly Inhomogeneous Medium for Very Long and Very Short Exposures. Journal of the Optical Society of America, v. 56, n. 10, p. 1372-1379, 1966.

GONZALEZ-CAVA, J. M. et al. ADRC: a strategy to deal with Adaptive Optics feedback control, Proceedings of the Adaptive Optics for Extremely Large Telescopes 5, 2017.

MEC. Diretrizes Curriculares Nacionais do Curso de Graduação em Engenharia. 2019. Disponível em: 〈http://portal.mec.gov.br/component/content/article?id=12991〉. Acesso em: 05 jan. 2021.

MAUCH, S. Robust Real-Time Control of an Adaptive Optics System. Tese de Doutorado Technische Universität IImenau, Fakultät für Informatik und Automatisierun, 2016.

OGATA, K. Engenharia de Controle Moderno. 5aㅗ edição, São Paulo: Pearson Prentice Hall, 2010.

NISE, N. S. Engenharia de Sistemas de Controle. 6ª edição, São Paulo: LTC, 2012.

RAPKIEWICZ, C. E.; FALKEMBACH, G. A. M.; SEIXAS, et al. Estratégias pedagógicas no ensino de algoritmos e programação associadas ao uso de jogos educacionais. RENOTE: Revista Novas Tecnologias na Educação, Rio Grande do Sul, v.4, n.2, p. 1-11, 2006.

REEVES, A. Soapy: an adaptive optics simulation written purely in Python for rapid concept development. Proceedings SPIE 9909, Adaptive Optics Systems V, v. 9909, p. 2173-2183, 2016.

OLIVIER, S. S.; MAX, C. E. Laser guide star adaptive optics: present and future [invited]. Proceedings of the 158th International Astronomical Union (IAU) Symposium, v. 158, p. 283-292, 1994.

\title{
Teaching Control Systems Using Adaptive Optics Concepts
}

\begin{abstract}
Several dynamic systems are presented to students in the control theory course to explain the concepts learned in real applications. Those systems have typically thermodynamic or electromechanical nature and are represented by first or second-order models. It is possible to increase students' ability to apply the concepts of modeling, simulation, and project controllers by presenting systems not commonly used in the classroom. This work presents three experiments of adaptive optics (AO). The first one consists of creating and setting simulation blocks of an AO system. The second one consists of projecting an Integral controller in the loop of the AO system created in the first experiment. The third experiment consists of applying a Proportional-Integral action controller. During all experiences, diagrams and graphs are generated allowing quantitative and qualitative discussions of the performance of the controllers. Thus, concepts of astronomical instrumentation are introduced, enabling students to exercise the concepts of control theory in an innovative context.
\end{abstract}

Keywords: Adaptive Optics. Astronomical Instrumentation. Process control. PID Controller. Engineering Teaching. 